\title{
Deep MRI Segmentation: A Convolutional Method Applied to Alzheimer Disease Detection
}

\author{
Hanane Allioui ${ }^{1}$, Mohamed Sadgal ${ }^{2}$, Aziz Elfazziki ${ }^{3}$ \\ Computer Science Department \\ Faculty of Sciences Semlalia \\ Cadi Ayyad University \\ Marrakech, Morocco
}

\begin{abstract}
The learning techniques have a particular need especially for the detection of invisible brain diseases. Learningbased methods rely on MRI medical images to reconstruct a solution for detecting aberrant values or areas in the human brain. In this article, we present a method that automatically performs segmentation of the brain to detect brain damage and diagnose Alzheimer's disease (AD). In order to take advantages of the benefits of $3 \mathrm{D}$ and reduce complexity and computational costs, we present a 2.5D method for locating brain inflammation and detecting their classes. Our proposed system is evaluated on a set of public data. Preliminary results indicate the reliability and effectiveness of our Alzheimer's Disease Detection System and demonstrate that our method is beyond current knowledge of Alzheimer's disease diagnosis.
\end{abstract}

Keywords-Computer-Assisted Diagnosis (CAD); Alzheimer's disease (AD); Image segmentation; Machine learning; Convolutional Neural Networks (CNN); Magnetic Resonance Imaging

\section{INTRODUCTION}

Alzheimer designates an incurable disease that attacks brain tissues and influences mental functions, as well as memory. This neurodegenerative disease is characterized by brain damage, including $\beta$-amyloid peptide $(A \beta)$, neurofibrillary tangles and neuronal degeneration, which chronically damage the brain in an irreversible way [1]. Scientific works on AD detection began around 1906 [2]. However, it took years to develop the appropriate algorithms to analyze information processing in the brain. Consequently, in the last decades, several systems based on CAD were proposed [3, 4]. However, most of the proposed systems have been based on manual functionalities requiring too much precision and concentration to study MRI modalities.

The automated Computer-Aided Diagnosis of Alzheimer remains significantly a challenging task that requires advanced technical practices such as Deep Learning (DL) algorithms. Recently, deep learning has shown promising methodologies with great progress in the segmentation, identification, and classification of image patterns [5, 6, 7]. Among the most widely used DL architectures, convolutional neural networks (CNNs) perform machine learning tasks without manual functions $[8,9]$. CNN has a strong ability to solve complex vision problems, such as classification $[10,11]$, segmentation [12] and object detection [13, 14].
The CNN network consists of different layers able to analyze and capture the quantity of the different structures present in the image. The extension of CNNs has contributed to the development of U-Net that allows the segmentation of biomedical images. The U-net is based entirely on a convolutional network, with a modified U-shaped architecture, which can perform the required tasks with fewer training images to produce more accurate segmentation swiftly using a GPU [15]. As for medical imaging, it is evident that U-Net architecture was introduced to promote precision and objects' localization in microscopic structures. U-Net combines a fully convolutional network [16] with a deconvolutional network [17]. Consequently, the resolution of the output can be ensured thanks to the number of features in the up-sampling phase, which ensures the propagation of context information to higher resolution layers.

This paper describes an improved CAD system for Alzheimer detection using $2.5 \mathrm{D}$ modalities. The ultimate contribution of our work is to provide a different Alzheimer detection process from several perspectives. First, the use of a robust neural network that can process the MRI scans on a 2.5D spatial context to improve the performance of segmentation and so that the whole system. Second, our method simultaneously performs brain structure segmentation for lesion detection and AD detection. Third, we tested our work on public data. Finally, we developed a novel 2.5D fully deep convoluted segmentation based on the U-Net architecture to increase the results accuracy.

This paper is ordered as follow: Section 2 states the literature review regarding Alzheimer detection techniques. Section 3 explains our proposed technique in detail. Section 4 illustrates the experimental results and Section 5 presents the conclusion of the work.

\section{LITERATURE REVIEW}

Practically, the diagnosis of $\mathrm{AD}$ is based on various essentials, such as genetic information, demographic factors, psychological and neuropsychological tests, indices of cerebrospinal fluid biomarkers (CSF) and brain imaging data [18]. Specifically, Numerous neuroimaging researches studied the region of interest (ROI) to inspect the brain changes caused by AD [19]. These works were based on prior knowledge to guide the selection of ROIs, which may neglect the consideration of brain changes outside of the studied areas. 
Otherwise, in [20] authors presented a spherical mapping based on 3D Brain images projection into 2D using statistical characteristics of brain tissue. Anitha et al. improved the watershed algorithm and presented a method to define the diseased area using shape analysis techniques. In the same way, Kalavathi et al. [21] used FFCM [22] to segment brain images whereas, machine-learning (ML) techniques offer the possibility of using systematic methods to ensure sophisticated, automatic classification and object-based detection [23]. The ML or more properly DL methods can learn from different models and manage complex changes in neuroimaging modalities, to ensure the analysis of large data with high accuracy.

During the last decades, the need for medical image processing has known explosive growth due to the development of technologies and the large-scale detected diseases especially the Alzheimer. Accordingly, intelligent vision systems have grown so fast. So, the understanding of brain images remains an area of rising interest in AD studies. Through the literature, different classification methods have shown good evidence. Dahshan et al. [24] used an artificial neural network (ANN) and K-nearest neighbor (KNN) networks for approximation extraction and detail coefficients calculation of the 3-level discrete wavelet transform (DWT). In [25]Park used learning for classification using two distance measurements which resulted in an error rate of $18 \%$ for the classification of $\mathrm{AD}$ cases and $46 \%$ for normal cases. Zang et al. [26] proposed a volumetric image classification method that implements a discrete 3D wavelet transform (3D-DWT) for wavelet coefficients extraction. Zhang et al. [27] presented an early $\mathrm{AD}$ detection based on Eigen brains and machine learning using SVM classifiers to detect accurately AD subjects as well as the AD-related brain regions.

With the advent of Computer Assisted Diagnosis systems, especially these, relying on deep learning techniques, The CNNs [28, 29] are frequently used to solve complicated problems from both computer vision and medical imaging field. Indeed, these models learn to establish a list of image characteristics. Since CNNs are much used for MRI images studies to detect or predict $\mathrm{AD}$, it is necessary to develop methods ensuring the learning from a large-scale training package [30]. The robustness of the Capsule or CapsNets [31] networks allows a fast, precise and thorough learning of data images. CapsNet requires less data for training by ensuring shorter learning curve [32]. Other methods have used different auto-encoders or CNN 3D to detect AD [33, 34].

Comparative evaluations were presented in [35], the authors presented a literature review of machine learning techniques used for $\mathrm{AD}$ detection. Their work illustrates the quality assessment of the previous studies and a comparison of the details. In addition, in recent years, the family of deep learning approaches has experienced considerable improvements, notably U-Net networks. Authors in [36] introduced a network that extends the u-net architecture from [37] by replacing all 2D processes with their 3D equivalent. Regarding brain segmentation, Chang et al. [38] proposed an effective 3D U-Net model to improve segmentation accuracy and feature labeling.
In contrast to all the previous solutions, the proposed method takes into consideration the incorporation of 3D spatial information in a $2.5 \mathrm{D}$ context using a deep convolutional network to ensure segmentation tasks. Our choice for U-Net is based on the need to boost the segmentation accuracy and the performance of image processing while studying a sensitive disease such as Alzheimer.

\section{PRoposed TECHNIQUE}

Taking into account the 2D and 3D modalities in image processing, we choose to adopt a $2.5 \mathrm{D}$ method which designates a set of techniques that take advantages of $3 \mathrm{D}$ features with fewer complexities. This involves extending the dimension of the lowest resolution of the MRI volume images into the RGB dimension providing different benefits:

- This polysemous approach enables a better representation of the 3-D features with lesser computational costs.

- The information of each slice of the MRI images can be exploited by transforming the 3D images into grayscale images with all the 3D information.

- The integration of 3D volumes can be achieved with less design and implementation requirements by optimizing memory, reducing complexity and ensuring flexibility.

\section{A. $3 D$ to $2.5 D$ Transformation and Data Preprocessing}

Our work aims AD by optimizing resources. For that, we have to cut the $3 \mathrm{D}$ image into a 2.5D image. Although the $2.5 \mathrm{D}$ concept was previously used in several works [39, 40], we used it to explore and characterize the emerging integration options between $2 \mathrm{D}$ and $3 \mathrm{D}$ brain images. The 2.5D is used in our work to present a sub-volume of dimensions $(x \times y \times z=3)$ where $x, y$, and $z$ are the MRI slice dimensions. Each 3D image contains several 2.5D scans, which cover the full studied area. To facilitate the implementation of our system, it is useful to take advantage of depth information (z-direction) in MRI images.

The point cloud $\mathrm{C}$ defines a set of points in a $3 \mathrm{D}$ volume like the example illustrated in Fig. 1, $\mathrm{C}$ can be written by $\mathrm{C}$ (xs, $y s, 3)$, where $\mathrm{s}$ is the spatial sampling step of the system and ( $\mathrm{x}$, y) are positive integers belonging to $\mathbb{Z} 2$. Therefore, the transformation of $\mathrm{C}$ into 2.5 projections can be performed without loss of data. Each point $\mathrm{C}$ (xs, ys, 3) will be converted to a pixel $\mathrm{P}(\mathrm{x}, \mathrm{y})$. Indeed, the value of the gray level of this pixel $\mathrm{P}$ will be $\mathrm{Z}-\mathrm{Zmin}$, where $\mathrm{Z}$ min is the minimum depth of C. Next, in volume 2.5 , the value of each pixel corresponds to the vertex of the point upper of Zmin. In contrast, the $2.5 \mathrm{~d}$ transformation in $3 \mathrm{D}$ corresponds to a simple conversion of the acquisition parameters to assemble the adjacent images and form the 3D volume.

Our method has been tested using public data from OASIS [41] which is an Open Access database containing several Series of imaging studies. The OASIS project has been initiated to ensure the availability of neuroimaging datasets to the scientific community. This database contains crosssectional T2 and longitudinal T1 datasets. We chose to use the 
T1w [42] data which was taken at the time of the disappearance of the transverse magnetization. These datasets have been used in previous studies concerning Alzheimer disease such as [43, 27]. In fact, the OASIS database includes 489 subjects ranging in age from 42 to 96 years old. However, our selection includes 126 cases after exclusion of cases whose information is not consistent as well as the patient's cases under the age of 60 years. The MRI images from the OASIS database have been pre-processed to ensure the quality of learning, so it is essential to standardize patient data. As described in [44, 45], the effects of normal aging have been removed from MRI images as they are likely to be similar to the effects caused by Alzheimer. This could cause problems of confusion regarding the estimation of the specificities of each case.
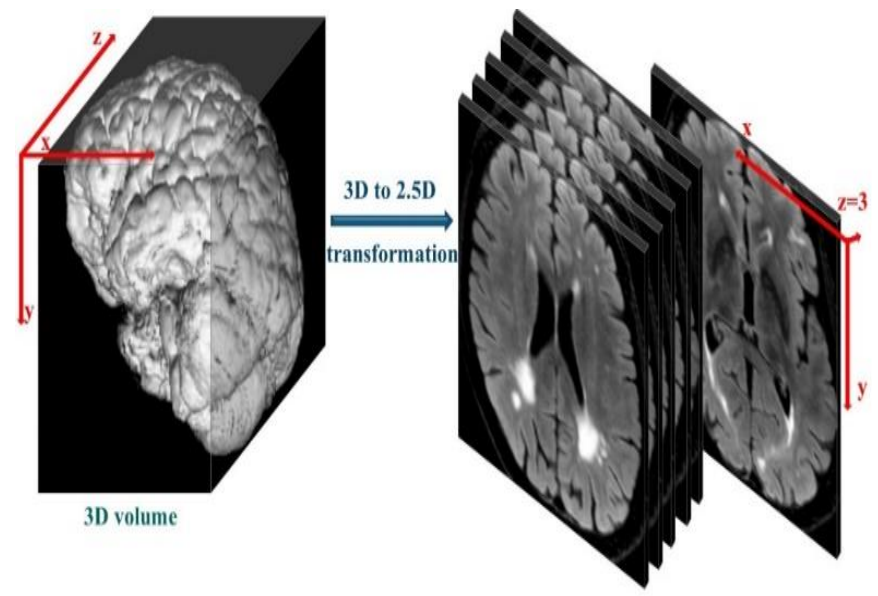

Set of adjacent $2.5 \mathrm{D}$ images

Fig. 1. Example of 3D to 2.5D Transformation.

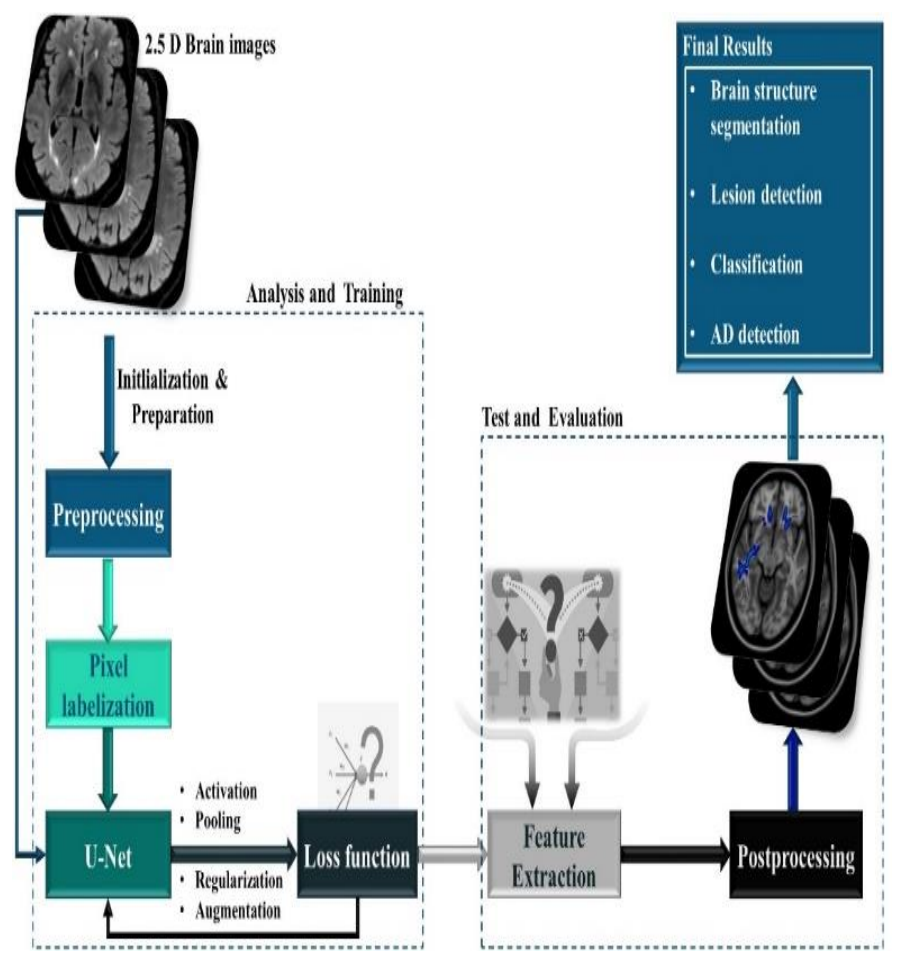

Fig. 2. Our Detailed Scheme of the Proposed CAD.

\section{B. The Proposed CAD Architecture}

In our system, the application of CNNs consists of convoluting a $2.5 \mathrm{D}$ image with the kernel in order to extract the maps of existing characteristics in the studied images. In fact, each map is connected to the previous layer of the network through the appropriate weights during the training to improve the input. The same kernel is converted on all the data of the image which leads to the use of several functions as will be explained in the following subsections. The proposed process is summarized in Fig. 2. We used the U-Net architecture for each view of the $2.5 \mathrm{D}$ brain MRI after parsing them into transversal views. The rationale behind the choice of this architecture is based on the sensitivity of the zones studied, and the limitation of memory facing the performed operations. Consequently, we have been able to optimize the formation of convolutional networks by limiting the problem of MRI images to a $2.5 \mathrm{D}$ domain.

Our method offers an AD diagnosis framework that extracts the characteristics of brain images, performs correct lesion detection, classifies their natures, and indicates the presence of $\mathrm{AD}$. The operations performed are designed to maximize the content of the information from different views. The details of this process are related to the following concepts:

- The initialization phase: it is the phase ensuring the convergence to launch the other tasks such as the activation and the regularization.

- The activation function which transforms an input to a set of outputs by imparting nonlinearity to the network structure. Thus, devoted to deep depreciation operations, the Rectifier linear units (ReLU) has demonstrated the acceleration of training while ensuring better results comparing with sigmoid functions [46].

- The Pooling phase allows the combination of neighboring entities spatially in feature maps. The redundancy of this combination provides a denser and less invariant representation for changes that may occur in the image and reduces the computational charges.

- The regularization reduces the overfitting by forcing all the nodes of the network to learn an improved representation of data and preventing nodes from coadapting to each other.

- The augmentation allows increasing the size of training sets and reducing the overfitting [47].

- The loss function defines the best optimal solution by determining the correct values for all weights. It's based on a learning model that minimizes the loss during the processing. For that reason, the loss function has to be minimized during the training.

In medical imaging, some tasks remain complicated such as labeling the learning images. This operation can be expensive and involve delicate and ambiguous decisions. For instance, in brain image processing, annotating the locations and scales of objects is often difficult, hence the need for a consistent way that annotates all the objects segment and detects their natures. 
In our article, we deal with a learning network to segment MRI images and form lists of the contained objects. As long as we rely on the U-Net architecture, we have introduced some changes. First, we introduce a function in the pooling layer that assumes the possible location of the segmented objects. Secondly, the cost function retrieves the information from the image and signals the location of objects or their distinctive part in the studied images.

1) Our U-Net Network architecture: We present our CAD system that leverages the power of $3 \mathrm{D}$ image processing by adopting a deep fully convolutional neural network, trained end-to-end. U-Net allowed us to ensure a precise sense of location. The goal of our architecture is to deal with brain image processing tasks to have an accurate analysis that can find out brain lesions, their localization as well as AD diagnosis. The U-net network has revealed promising results on biomedical images [48] and natural images [49].

Fig. 3 outlines our U-Net network. Our CAD system is based on the U-Net which includes a contracting (downsampling) path and an expanding (up-sampling) path.

The contracting path consists of 5 convolution blocks that each of them contains two convolutional layers of size $(3 \times 3)$, a stride of 1 in addition to a rectifier activation which makes it possible to increase the number of cards from 1 to 1024 . Except for the last block, by the end of each block, the maxpooling $(2 \times 2)$ is applied. In the expanding path, each of the blocks begins with deconvolution of size $(3 \times 3)$ in addition to a stride of $(2 \times 2)$. Indeed, each convolutional layers of the contracting part (encoder) has a corresponding decoder in the deconvolutional layers. Finally, we opted for a SoftMax layer. Then at the end of our network a classification layer to determine the classes of the detected objects in the studied brain images.

2) Training and optimization: Once we have completed the design of our network, tested the structure we start the training process. For that, extracting global characteristics of MRI images requires large training and involves costly computation, given a large number of test parameters crossing the input/output layers. To evaluate our 2.5D approach, the Soft Dice metric was used as a network cost function during the formation phase, this metric representing a differentiable form of the DSC (DSC) [50]. For better efficiency, in the processing of MRI images, optimization based on a stochastic gradient is essential during the training phase to minimize the cost function according to its parameters. After training, the intensity is normalized by providing a linear transformation of the original intensities between two features into the corresponding learned ones. This allows the similarity of the histogram of each sequence between subjects. The time required was of the order of ten minutes.

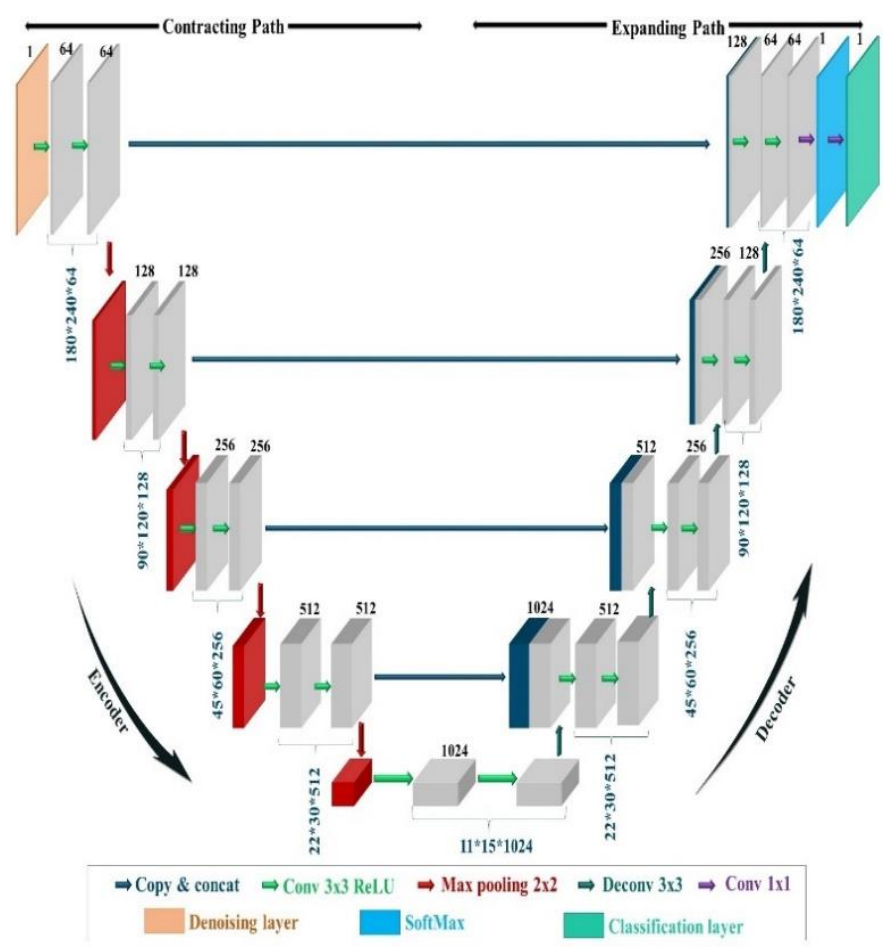

Fig. 3. Our U-Net Architecture.

\section{EXPERIMENTS AND RESULTS}

\section{A. Experiments and Performance Evaluation}

In this paper, we have proposed and developed a CAD system using deep convolutional networks to analyze brain damage and detect Alzheimer's disease by segmenting 2.5D images. As long as we have added a classification layer in our network, we need accurate measures to calculate the proportional values between the studied samples. For this purpose, we considered three metrics: accuracy, sensitivity, and specificity $[51,52]$. These metrics are defined as:

Accuracy $=\frac{\mathrm{TP}+\mathrm{TN}}{\mathrm{TP}+2 \mathrm{TN}+\mathrm{FP} \prime}$

Sensitivity $=\frac{\mathrm{TP}}{\mathrm{TP}+\mathrm{FN} \prime}$

Specificity $=\frac{\mathrm{TN}}{\mathrm{TN}+\mathrm{FP}}$

Where:

- TP represents the value of the true positive (the number of cases having an $\mathrm{AD}$ correctly).

- $\mathrm{TN}$ is the true negative (the number of control cases)

- FP represents the value of the false positive (the number of control cases with AM)

- $\mathrm{FN}$ is the false negative value (the number of cases with AD considered as controls). 


\section{B. Results and Discussion}

As shown in Fig. 3, our method involves of a fully CNN containing two main parts: The U-net to segment the brain images and the classification layer to classify the detected areas. The input image will first go through the contracting path to generate feature maps, then the expanding path to generate the output segmented image. After that, the detected objects in the segmented images would be classified to detect their nature and improve the network model's adaptability.

In the first experiment, height hundred images are used in order to train and validate our model. An example of the results is shown in Fig. 4 which illustrates the detection and segmentation of endometrial areas of the brain are part of the semantic segmentation. After the training phase, we carried out different sets of evaluations. This allowed us to add new examples to our initial training. In this phase, we compared the segmentation result with the classification of detected objects in cerebral images. The network can detect cases of brain damage or lesion as shown in case a. They may affect brain abilities and cause different problems. In these cases, the patient may have similar signs similar to those of AD, yet these lesions can be treated. Case $b$ shows both brain damage and Alzheimer disease presence in the early stages. Finally, case c denotes the clear presence of $\mathrm{AD}$, which shows a big difference comparing to healthy aged brains.

Currently, there is no perfect available method for image segmentation and object detection. For performance verification, the experimental evaluation is performed using a series of different previous methods. In the second experiment, we tested the results of our developed network, as well as different methods which don't need a prior training domain were known, are evaluated. All these methods are based on convolutional structures. Hosseini et al. [53] presented an adapted 3D Convolutional Network to predict the AD and proved competitive results. However, this work was dedicated to only image classification for $3 \mathrm{D}$ images. This indicates the need to adapt the network if we want to process $2.5 \mathrm{D}$ images to have high efficiency and update some functions to ensure image annotation concerning the segmented areas. Kalavathi et al. [21] proposed a method based on segmentation of the brain using contours to eliminate the area of the hug, then they applied Fast Fuzzy C Means (FFCM) to segment brain tissue to detect the existence of Alzheimer's disease. Hao Dong et al. [54] presented a reliable method for automatically segmenting brain tumors. They used deep U-Net convolution networks. They had good results for the detection of tumor regions. Yet they don't take into consideration brain damages without AD presence.

As illustrates the example in Fig. 5 the results demonstrate the efficacy of our method. Another important outcome is the number of wrong region detection without the classification stage. The methods in Fig. 5(b) and Fig. 5(d) show good results, however they can segment other objects different from the zones attacked by the Alzheimer which can create a kind of confusion during the diagnosis. In Fig. 5(c) the method ensures the segmentation of the brain by determining different objects other than lesions or $\mathrm{AD}$. This phenomenon is mainly due to the fact that even the semantic segmentation is not only sufficient to diagnose AD. That's why it's preferable to combine several techniques to achieve higher accuracy.

Compared to the quantitative results of the different mentioned methods, the validation of the proposed system demonstrated good results for the complete brain segmentation. Using our method allows us to achieve an Accuracy rate of $92.71 \%$, Sensitivity of $94.43 \%$, and a Specificity rate of $91.59 \%$.

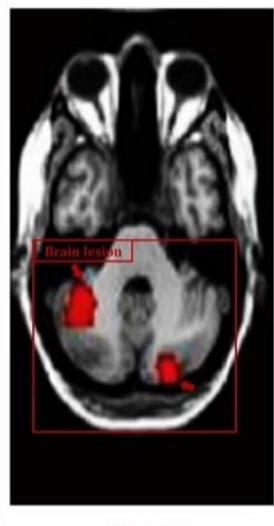

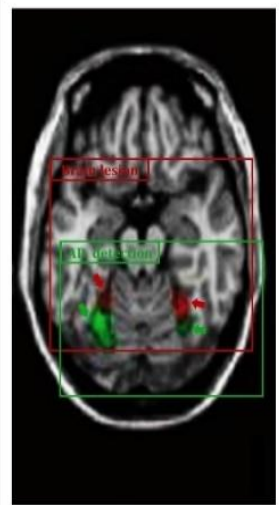

Case (b)

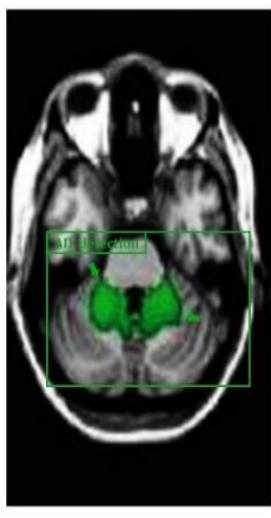

Case (c)
Fig. 4. Example of Possible Results [ Case (a): Presence of many Lesions that may Affect Brain Function; Case (b): Significant Loss of different Brain Areas, with Lesions Present (Red Color), Plus an Onset of Alzheimer's Disease (Green Color); Case (c): Strong Presence of AD Compared to Healthy Patients.].
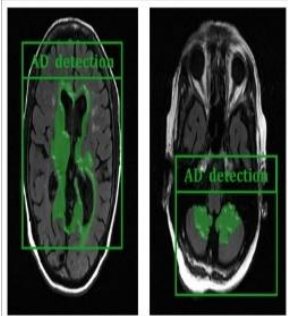

(a)
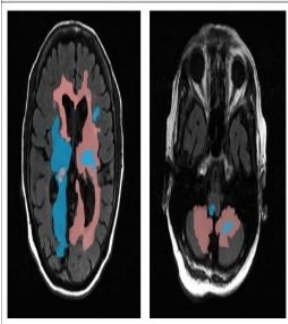

(b)
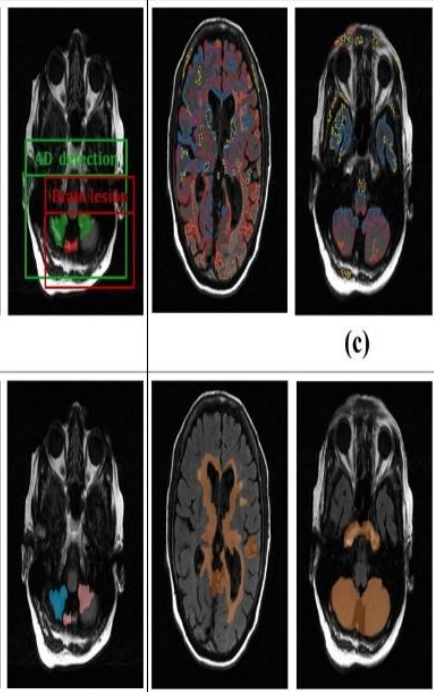

(d)
Fig. 5. Example of the different Tested Methods Results [ a-Our Proposed Method, b- Hao Dong [54], Kalavathi et al. [21], Hosseini et al. [53]].

TABLE. I. QuANTITATIVE Results OF OUR PROPOSED Method COMPARED TO THE RESULTS FROM OTHER METHODS

\begin{tabular}{|l|l|l|l|}
\hline Tested method & Accuracy & Sensitivity & Specificity \\
\hline a-Our method & $\mathbf{9 2 . 7 1} \%$ & $\mathbf{9 4 . 4 3 \%}$ & $\mathbf{9 1 . 5 9 \%}$ \\
\hline b- Hao Dong [54] & $89.75 \%$ & $85.85 \%$ & $87.34 \%$ \\
\hline c- Kalavathi et al. [21] & $55.83 \%$ & $56.28 \%$ & $51.11 \%$ \\
\hline d- Hosseini et al. [53] & $90.31 \%$ & $91.18 \%$ & $90.51 \%$ \\
\hline
\end{tabular}


To quantitatively assess the effectiveness of the methods tested we present, in a random order, the results of the evaluation methods as shown in Table I. To ensure better comparison we used the same datasets from Oasis to assess them. we conclude that the perfect segmentation of cerebral images is not granted by any method. However, the proposed method was able to take first place in terms of segmentation performance taking into account the use of the same evaluation basis. According to these results, we report the high-efficiency of our proposed network.

In our process, firstly the down-sampling and training are made. Then the up-sampling outputs are classified. Visually the results indicate that our proposal can segment brain images to diagnose $\mathrm{AD}$; the task that requires physician's interactions in the general clinical routine; by offering blended annotation on the studied images. Moreover, our quantitative results reveal further efficiency with the additional classification layer. This layer facilitates $\mathrm{AD}$ detection with less additional efforts.

The proposed network offers accurate results in less time (less than 2 hours for training and testing). These accomplishments are superiors than all the tested methods that can take more hours or even days for brain images analysis. Our method is an appropriate automatic solution for brain image segmentation and $\mathrm{AD}$ detection. Yet, the current work still has some limitations. First, our method was evaluated to segment 2.5D images using a T1 longitudinal dataset, but running our method on a t 2 dataset can produce a more objective evaluation. Secondly, the evolution of neural networks continues to increase, so the addition of new parameters can improve the performed tasks by our network.

\section{CONCLUSION}

In this work, we proposed a developed U-Net architecture for brain images segmentation intended for Alzheimer disease and brain damage detection. We found-out that U-Net improves clearly state-of-the-art. The benefits of our method were demonstrated in comparison to different relevant methods. Our main contribution is providing an automatic and exact Alzheimer detection using an advanced full neural network on a $2.5 \mathrm{D}$ context to ensure the performance of segmentation whatever the used system, by reducing memory costs while processing 3D images. Moreover, our method performs brain segmentation then classification for $\mathrm{AD}$ detection. To our best knowledge, our proposed CAD system is the first one 2.5D MRI analysis for Alzheimer's disease detection using such learning techniques. Our developed U-Net can automatically segment a 2.5D MRI image and offer an accurate analysis of brain structures. The network to segment brain images was trained from scratch, for that we expect that it will be applicable to many brain analyses especially $2.5 \mathrm{D}$ segmentation tasks.

Our deep U-Net network has been able to obtain competitive results in detecting damaged regions of the brain and defining the presence of $\mathrm{AD}$. The proposed method generates a model of automatic segmentation of brain lesions as well as the diagnosis of patient-specific $\mathrm{AD}$, which can potentially facilitate different clinical tasks such as diagnosis, treatment planning, and patient monitoring.

\section{REFERENCES}

[1] J. Sevigny et al., "The antibody aducanumab reduces $A \beta$ plaques in Alzheimer's disease", Nature, vol. 537, pp. 50-56, 2016.

[2] NC. Berchtold and CW. Cotman, "Evolution in the conceptualization of dementia and Alzheimer's disease", Neurobiology of Aging. vol. 19, n. 3, pp. 173-189, 1998.

[3] R. Chaves et al., "SVM-based computer-aided diagnosis of the Alzheimer's disease using t-test NMSE feature selection with feature correlation weighting", Neuroscience Letters, vol. 461, pp. 293-297, 2009.

[4] E. Bron et al., "Standardized evaluation of algorithms for computer aided diagnosis of dementia based on structural MRI: the CAD Dementia challenge", Neuroimage, vol. 111, pp. 562-579, 2015.

[5] F. Li et al., "A Robust Deep Model for Improved Classification of AD/MCI Patients", IEEE Journal of Biomedical and Health Informatics, vol. 19,n. 5,pp. 1610 - 1616, 2015.

[6] N. Zeng et al., "Facial expression recognition via learning deep sparse autoencoders", Neurocomputing, vol. 273, pp. 643-649, 2018.

[7] W. Lin et al., "Convolutional Neural Networks-Based MRI Image Analysis for the Alzheimer's Disease Prediction From Mild Cognitive Impairment" Frontiers in Neuroscience, vol. 12, n. 777, 2018.

[8] D. Nie et al., "Fully convolutional networks for multi-modality isointense infant brain image segmentation", Procedding of the 2016 IEEE International Symposium on Biomedical Imaging (ISBI), pp. 1342-1345, 2016.

[9] H. C. Shin et al., "Deep Convolutional Neural Networks for Computer Aided Detection: CNN Architectures, Dataset Characteristics and Transfer Learning", IEEE Transactions on Medical Imaging, vol. 35, n.5, pp. 1285-1298, 2016.

[10] A. Krizhevsky et al., "Imagenet classification with deep convolutional neural networks", Advances in Neural Information Processing Systems, pp. 1097-1105, 2012.

[11] C. Szegedy et al., "Going deeper with convolutions", The IEEE Conference on Computer Vision and Pattern Recognition, pp. 1-9, 2015.

[12] K. Kamnitsas et al., "Efficient multi-scale 3D CNN with fully connected CRF for accurate brain lesion segmentation", Medical Image Analysis, vol. 36, pp. 61-78, 2017.

[13] C. Szegedy et al., "Deep neural networks for object detection", Advances in Neural Information Processing Systems, vol. 26, pp. 25532561, 2013.

[14] P. Sermanet et al., "OverFeat: Integrated Recognition, Localization and Detection using Convolutional Networks", Computer Vision and Pattern Recognition, 2014.

[15] M. Beyeler et al., "A GPU-accelerated cortical neural network model for visually guided robot navigation”, Neural Networks, vol. 72, pp. 75-87, 2015.

[16] J. Long et al., "Fully convolutional networks for semantic segmentation", Proceedings of the IEEE 2015 Conference on Computer Vision and Pattern Recognition, pp. 3431-3440, 2015.

[17] H. Noh et al., "Learning deconvolution network for semantic segmentation", The Proceedings of the IEEE 2015 International Conference on Computer Vision, pp. 1520-1528, 2015.

[18] A. Sarica et al., "Random Forest Algorithm for the Classification of Neuroimaging Data in Alzheimer's Disease: A Systematic Review", Frontiers in Aging Neuroscience, vol. 9, n. 329, 2017.

[19] JP. Lerch et al., "Automated cortical thickness measurements from MRI can accurately separate Alzheimer's patients from normal elderly controls", Neurobiol Aging, vol. 29, n. 1, pp. 23-30, 2008.

[20] FJ. Martinez-Murcia et al., "A Spherical brain mapping of MR images for the dectection of Alzheimer's disease", Current Alzheimer Research, vol. 13, n. 5, pp. 575-588, 2016.

[21] P. Kalavathi et al., "Detection of Alzheimer Disease in MR Brain Images using FFCM Method", Computational Methods, Communication Techniques and Informatics, 2017.

[22] W. Cai et al., "Fast and robust fuzzy c-means clustering algorithms incorporating local information for image segmentation", Pattern Recognition, vol. 40, n. 3, pp. 825-838, 2007. 
[23] P. Sajda, "Machine learning for detection and diagnosis of disease", Annual Review of Biomedical Engineering, vol. 8, pp. 537-65, 2006.

[24] E. A. El-Dahshan et al., "Hybrid intelligent technique for MRI brain images classification”, Digital Signal Processing, vol. 20, n. 2, pp. 433441, 2010.

[25] H.J. Park, "Comparison of distance measures for manifold learning: Application to Alzheimer's brain scans", Journal of the Korean Physical Society, vol. 61, n. 7, pp. 1148-1155, 2012.

[26] Y. Zhang et al., "Detection of Alzheimer's disease and mild cognitive impairment based on structural volumetric MR images using 3D-DWT and WTA-KSVM trained by PSOTVAC", Biomedical Signal Processing and Control, vol. 21, pp. 58-73, 2015.

[27] Y. Zhang et al., "Detection of subjects and brain regions related to Alzheimer's disease using 3D MRI scans based on eigenbrain and machine learning", Frontiers in Computational Neuroscience, vol. 9, n.66, 2015.

[28] D. B. Sam et al., "Divide and Grow: Capturing Huge Diversity in Crowd Images with Incrementally Growing CNN", The IEEE Conference on Computer Vision and Pattern Recognition (CVPR), pp. 3618-3626, 2018.

[29] C. Zhang et al., "VPRS-based regional decision fusion of CNN and MRF classifications for very fine resolution remotely sensed images", IEEE Transactions on Geoscience and Remote Sensing, vol. 56, n. 8, pp. 4507 - 4521, 2018.

[30] K.R. Kruthik et al., "CBIR system using Capsule Networks and 3D CNN for Alzheimer's disease diagnosis", Informatics in Medicine Unlocked, vol. 14, pp. 59-68, 2019.

[31] S. Sabour et al., "Dynamic routing between capsules", Advances in Neural Information Processing Systems, pp. 3856-3866, 2017.

[32] D. E. Worrall et al., "Harmonic Networks: Deep Translation and Rotation Equivariance", IEEE Conference on Computer Vision and Pattern Recognition IEEE Computer Society, pp. 7168-7177, 2017.

[33] J. Liu et al., "Alzheimer's disease classification based on individual hierarchical networks constructed with 3-D texture features", IEEE Trans Nano Bioscience, vol. 16, n. 6, pp. 428-437, 2017.

[34] S. Liu et al., "Early diagnosis of Alzheimer's disease with deep learning", IEEE 11th International Symposium on Biomedical Imaging, pp. 1015-1018, 2014.

[35] E. Pellegrini et al., "Machine learning of neuroimaging to diagnose cognitive impairment and dementia: a systematic review and comparative analysis", arXiv:1804.01961, 2018.

[36] Ö. Çiçek et al., "3D U-Net: Learning Dense Volumetric Segmentation from Sparse Annotation", MICCAI 2016: Medical Image Computing and Computer Assisted Intervention, pp. 424-432, 2016.

[37] O. Ronneberger et al., "U-Net: convolutional networks for biomedical image segmentation”, MICCAI 2015. LNCS, n. 9351, pp. 234-241, Springer, Heidelberg, doi: 10.1007/97, 2015.

[38] J. Chang et al., "Brain Tumor Segmentation Based on 3D Unet with Multi-Class Focal Loss", The 2018 International Congress on Image and Signal Processing, BioMedical Engineering and Informatics (CISPBMEI), DOI: 10.1109/CISP-BMEI.2018.8633056, 2018.
[39] H. R. Roth et al., "A new $2.5 \mathrm{~d}$ representation for lymph node detection using random sets of deep convolutional neural network observations", The International Conference on Medical Image Computing and Computer-Assisted Intervention, Springer, pp. 520-527, 2014.

[40] H. R. Roth et al., "A New 2.5D Representation for Lymph Node Detection using Random Sets of Deep Convolutional Neural Network Observations", Medical Image Computing and Computer-Assisted Intervention, vol. 17, n. 1, pp. 520-527, 2014.

[41] Open Access Series of Imaging Studies. [Online] 2010. https://www.oasis-brains.org.

[42] B. A. Ardekani et al., "Corpus callosum shape changes in early Alzheimer's disease: an MRI study using the OASIS brain database", Brain Structure and Function, vol. 219, pp. 343-352, 2014.

[43] A. B. Tufail et al., "Multiclass classification of initial stages of Alzheimer's disease using structural MRI phase images", IEEE International Conference on Control System, Computing and Engineering, pp. $23-25,2012$.

[44] J. Dukart et al., "Age correction in dementia matching to a healthy brain", Alzheimer's Disease Neuroimaging Initiative, PLoS One, vol. 6, n.7, 2011.

[45] E. Moradi et al., "Machine learning framework for early MRI-based Alzheimer's conversion prediction in MCI subjects", Neuroimage, vol. 104, pp. 398-412, 2015.

[46] K. Jarrett et al., "What is the best multi-stage architecture for object recognition", International Conference IEEE Computer Vision, pp. 2146-2153, 2009.

[47] A. Krizhevsky et al., "Imagenet classification with deep convolutional neural networks", Advances in Neural Information Processing Systems, pp. 1097-1105, 2012.

[48] M. Drozdzal et al., "The Importance of Skip Connections in Biomedical Image Segmentation", Deep Learning and Data Labeling for Medical Applications, pp. 179-187, 2016.

[49] J. Long et al., "Fully convolutional networks for semantic segmentation", IEEE Conference on Computer Vision and Pattern Recognition (CVPR), pp. 3431-3440, 2015.

[50] F. Milletari et al., "V-Net: Fully Convolutional Neural Networks for Volumetric Medical Image Segmentation”, International Conference on 3D Vision (3DV), 2016.

[51] M. Haubro et al., "Sensitivity and specificity of CT- and MRI-scanning in evaluation of occult fracture of the proximal femur", Injury, vol. 46, n.8, pp. 1557-1561, 2015.

[52] U. A. Hashim et al., "Diagnostic accuracy of multi-parametric MRI and TRUS biopsy in prostate cancer (PROMIS): a paired validating confirmatory study", The Lancet, vol. 389, n. 10071, pp. 815-822, 2017.

[53] E. Hosseini-Asl et al., "Alzheimer's Disease Diagnostics by Adaptation of 3D Convolutional Network", IEEE International Conference on Image Processing, 2016.

[54] H. Dong et al., "Automatic Brain Tumor Detection and Segmentation Using U-Net Based Fully Convolutional Networks", MIUA: Medical Image Understanding and Analysis, pp. 506-517, 2017. 